\title{
The Nonparametric Approach to Demand Analysis
}

\author{
Hal R. Varian \\ Econometrica, Vol. 50, No. 4. (Jul., 1982), pp. 945-973.
}

Stable URL:

http://links.jstor.org/sici?sici=0012-9682\%28198207\%2950\%3A4\%3C945\%3ATNATDA\%3E2.0.CO\%3B2-O

Econometrica is currently published by The Econometric Society.

Your use of the JSTOR archive indicates your acceptance of JSTOR's Terms and Conditions of Use, available at

http://www.jstor.org/about/terms.html. JSTOR's Terms and Conditions of Use provides, in part, that unless you have obtained prior permission, you may not download an entire issue of a journal or multiple copies of articles, and you may use content in the JSTOR archive only for your personal, non-commercial use.

Please contact the publisher regarding any further use of this work. Publisher contact information may be obtained at http://www.jstor.org/journals/econosoc.html.

Each copy of any part of a JSTOR transmission must contain the same copyright notice that appears on the screen or printed page of such transmission.

The JSTOR Archive is a trusted digital repository providing for long-term preservation and access to leading academic journals and scholarly literature from around the world. The Archive is supported by libraries, scholarly societies, publishers, and foundations. It is an initiative of JSTOR, a not-for-profit organization with a mission to help the scholarly community take advantage of advances in technology. For more information regarding JSTOR, please contact support@jstor.org. 


\title{
THE NONPARAMETRIC APPROACH TO DEMAND ANALYSIS
}

\author{
BY HAL R. VARIAN ${ }^{1}$
}

\begin{abstract}
This paper shows how to test data for consistency with utility maximization, recover the underlying preferences, and forecast demand behavior without making any assumptions concerning the parametric form of the underlying utility or demand functions.
\end{abstract}

THE ECONOMIC THEORY of consumer demand is extremely simple. The basic behavioral hypothesis is that the consumer chooses a bundle of goods that is preferred to all other bundles that he can afford. Applied demand analysis typically addresses three sorts of issues concerning this behavioral hypothesis.

(i) Consistency. When is observed behavior consistent with the preference maximization model?

(ii) Recoverability. How can we recover preferences given observations on consumer behavior?

(iii) Extrapolation. Given consumer behavior for some price configurations how can we forecast behavior for other price configurations?

The standard approach to these questions proceeds by postulating parametric forms for the demand functions and fitting them to observed data. The estimated demand functions can then be tested for consistency with the maximization hypothesis, used to make welfare judgements, or used to forecast demand for other price configurations. This procedure will be satisfactory only when the postulated parametric forms are good approximations to the "true" demand functions. Since this hypothesis is not directly testable, it must be taken on faith.

In this paper I describe an alternative approach to the above problems in consumer demand analysis. The proposed approach is nonparametric in that it requires no ad hoc specifications of functional forms for demand equations. Rather, the nonparametric approach deals with the raw demand data itself using techniques of finite mathematics. In particular I will show how one can directly and simply test a finite body of data for consistency with preference maximization, recover the underlying preferences in a variety of formats, and use them to extrapolate demand behavior to new price configurations. Thus each of the issues of concern to demand analysis mentioned above is amenable to the nonparametric approach. ${ }^{2}$

\section{TESTING FOR CONSISTENCY WITH THE MAXIMIZATION HYPOTHESIS}

Let $p^{l}=\left(p_{1}^{i}, \ldots, p_{k}^{l}\right)$ denote the $i$ th observation of the prices of some $k$ goods and let $x^{i}=\left(x_{1}^{i}, \ldots, x_{k}^{i}\right)$ be the associated quantities. Suppose that we have $n$

\footnotetext{
${ }^{1}$ This work was financed by grants from the National Science Foundation and the Guggenheim Memorial Foundation. I wish to thank Erwin Diewert, Avinash Dixit, Joseph Farrell, Angus Deaton, and Sydney Afriat for comments on an earlier draft.

${ }^{2}$ Another concern of applied demand analysis is the issue of testing for restrictions on the form of the utility function or budget constraint such as homotheticity, separability, etc. I address these questions in Varian [29, 30].
} 
observations on these prices and quantities, $\left(p^{i}, x^{i}\right), i=1, \ldots, n$. How can we tell if these observations could have been generated by a neoclassical, utility maximizing consumer?

DefinItIon: A utility function $u(x)$ rationalizes a set of observations $\left(p^{i}, x^{i}\right)$, $i=1, \ldots, n$, if $u\left(x^{i}\right) \geqq u(x)$ for all $x$ such that $p^{i} x^{i} \geqq p^{i} x$.

At the most general level there is a very simple answer to the above question: any finite number of observations can be rationalized by the trivial constant utility function $u(x)=1$ for all $x$. The real question is when can the observations be rationalized by a sufficiently well behaved nondegenerate utility function? The best results in this direction are due to Sydney Afriat $[1,2,3,4,5]$.

AfriaT'S THEOREM: The following conditions are equivalent:

(1) There exists a nonsatiated utility function that rationalizes the data.

(2) The data satisfies "cyclical consistency"; that is,

$$
p^{r} x^{r} \geqq p^{r} x^{s}, \quad p^{s} x^{s} \geqq p^{s} x^{t}, \quad \ldots, \quad p^{q} x^{q} \geqq p^{q} x^{r}
$$

implies

$$
p^{r} x^{r}=p^{r} x^{s}, \quad p^{s} x^{s}=p^{s} x^{t}, \quad \ldots, \quad p^{q} x^{q}=p^{q} x^{r} .
$$

(3) There exist numbers $U^{i}, \lambda^{i}>0, i=1, \ldots, n$, such that

$$
U^{i} \leqq U^{j}+\lambda^{j} p^{j}\left(x^{i}-x^{j}\right) \quad \text { for } i, j=1, \ldots, n .
$$

(4) There exists a nonsatiated, continuous, concave, monotonic utility function that rationalizes the data.

\section{Proof: See Appendix 1.}

There are several remarkable features of Afriat's theorem. First, the equivalence of (1) and (4) shows that if some data can be rationalized by any nontrivial utility function at all it can in fact be rationalized by a very nice utility function. Or put another way, violations of continuity, concavity, or monotonicity cannot be detected with only a finite number of demand observations. Secondly, the numbers $U^{i}$ and $\lambda^{i}$ referred to in part (3) of Afriat's theorem can be used to actually construct a utility function that rationalizes the data. The numbers $U^{i}$ and $\lambda^{i}$ can be interpreted as measures of the utility level and marginal utility of income at the observed demands. This is described in more detail in Appendix 1.

Thirdly, parts (2) and (3) of Afriat's theorem give directly testable conditions that the data must satisfy if it is to be consistent with the maximization model. Condition (3) for example simply asks whether there exists a nonnegative solution to a set of linear inequalities. The existence of such a solution can be checked by solving a linear program with $2 n$ variables and $n^{2}$ constraints. Diewert and Parkan [10] describe some of their computational experience with this technique using actual demand data. Unfortunately the fact that the number 
of constraints rises as the square of the number of observations makes this condition difficult to verify in practice for computational reasons. ${ }^{3}$

Condition (2) seems rather more promising from the computational perspective. As it turns out, there is an equivalent formulation of condition (2) which is quite easy to test. In addition this equivalent formulation is much more closely related to the traditional literature on the revealed preference approach to demand theory of Samuelson [24], Houthakker [12], Richter [21], and others. In order to describe this formulation we must first consider the following definitions:

Definitions: Given an observation $x^{i}$ and a bundle $x$ :

(1) $x^{i}$ is directly revealed preferred to $x$, written $x^{i} R^{0} x$, if $p^{i} x^{i} \geqq p^{i} x$.

(2) $x^{i}$ is strictly directly revealed preferred to $x$, written $x^{i} P^{0} x$, if $p^{i} x^{i}>p^{i} x$.

(3) $x^{i}$ is revealed preferred to $x$, written $x^{i} R x$, if $p^{i} x^{i} \geqq p^{i} x^{j}, p^{j} x^{j}$ $\geqq p^{j} x^{l}, \ldots, p^{m} x^{m} \geqq p^{m} x$ for some sequence of observations $\left(x^{i}, x^{j}, \ldots, x^{m}\right)$. In this case we say that the relation $R$ is the transitive closure of the relation $R^{0}$.

(4) $x^{i}$ is strictly revealed preferred to $x$, written $x^{\prime} P x$, if there exist observations $x^{j}$ and $x^{l}$ such that $x^{i} R x^{j}, x^{j} P^{0} x^{l}, x^{l} R x$.

Note that in the above definitions we do not require $x^{\prime}, x^{j}, x^{l}$, etc. to be distinct observations. We also adopt the convention that $x R x$ for all bundles $x$.

DEFINITIONS: A set of data satisfies the:

(1) Strong Axiom of Revealed Preference, version 1 (SARP 1) if $x^{i} R x^{\prime}$ and $x^{J} R x^{\prime}$ implies $x^{i}=x^{j}$

(2) Strong Axiom of Revealed Preference, version 2 (SARP 2) if $x^{i} R x^{j}$ and $x^{i} \neq x^{j}$ implies not $x^{j} R x^{i}$;

(3) Strong Axiom of Revealed Preference, version 3 (SARP 3) if $x^{\prime} R x^{j}$ and $x^{i} \neq x^{J}$ implies not $x^{j} R^{0} x^{i}$;

(4) Generalized Axiom of Revealed Preference (GARP) if $x^{i} R x^{j}$ implies not $x^{j} P^{0} x^{i}$.

The most common statement of the Strong Axiom is probably SARP $2 .{ }^{4}$ It is clear that SARP 1 is equivalent to SARP 2. It is not quite so clear that SARP 3 is equivalent to SARP 2, but nevertheless they are equivalent. One can easily show that SARP 1, SARP 2, and SARP 3 imply GARP, but not vice versa. Basically SARP (in any of its formulations) requires single valued demand functions while GARP is compatible with multivalued demand functions. For example, the data in Figure 1 violate SARP but are quite compatible with GARP.

\footnotetext{
${ }^{3}$ One can always use the duality theorem of linear programming to construct an equivalent problem with $n^{2}$ variables and $2 n$ constraints, but this problem may also be computationally difficult.

${ }^{4}$ See Richter [22] for several variations on revealed preference axioms. Note that Richter considers a framework where the entire demand correspondence is given, rather than only a finite number of observations. This leads to a number of differences in the analysis.
} 


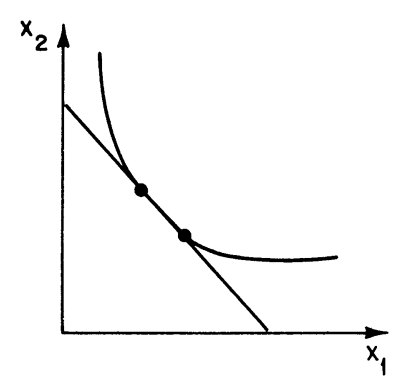

Figure 1.

This is why we refer to GARP as the Generalized Axiom of Revealed Preference. It turns out to be a necessary and sufficient condition for data to be consistent with utility maximization, and is in fact equivalent to Afriat's cyclical consistency condition.

FACT 1: A set of data satisfies cyclical consistency if and only if it satisfies GARP.

Proof: Suppose that we have some data containing a violation of cyclical consistency so that $p^{r} x^{r} \geqq p^{r} x^{s}, \ldots, p^{j} x^{j}>p^{j} x^{i}, \ldots, p^{q} x^{q} \geqq p^{q} x^{r}$. Then $x^{i} R x^{j}$ by going around the cycle, and $x^{j} P^{0} x^{i}$ directly. Hence we have a violation of GARP.

On the other hand, suppose we have some data that has a violation of GARP. Then writing out the violation in the above form shows we have a violation of cyclical consistency also.

The equivalency of GARP and cyclical consistency is trivial from the mathematical point of view, but is quite important from the computational point of view, since GARP is quite simple to check in practice, as we discuss below.

First, let us note that GARP can be restated as: if $x^{i} R x^{j}$ then $p^{j} x^{j} \leqq p^{j} x^{i}$ for $i, j=1, \ldots, n$. Hence verifying that some data satisfies GARP is trivial once we know the relation $R$-the transitive closure of the direct revealed preference relation $R^{0}$.

It is clear that the computation of the transitive closure of a finite relation is a finite problem. The only issue is how one might compute it efficiently. This question has been addressed in the economics literature by Koo $[14,15,16]$, Dobell [7], and Uebe [28], and in the computer science literature by Warshall [31] and Munroe [20], among others.

Most of the algorithms in the economics literature compute the transitive closure of a relation in time proportional to $n^{4}$. The computer scientists, utilizing the law of comparative advantage, do a bit better. Warshall's algorithm computes the transitive closure in $n^{3}$ steps, and Munroe describes a process that does it in time proportional to $n^{2.74}$. Warshall's algorithm is especially easy to implement 
and quite ingenious. It seems fast enough for the problems encountered in economics, as well. We therefore describe Warshall's algorithm in Appendix 2.

At this point it might be worthwhile to be rather explicit about how one represents the relations $R^{0}$ and $R$ in a form suitable for computation and how one actually verifies GARP in a systematic way.

Let us construct an $n$ by $n$ matrix $M$ whose $i-j$ entry is given by:

$$
m_{i j}= \begin{cases}1 & \text { if } p^{i} x^{i} \geqq p^{i} x^{j}, \text { that is, } x^{i} R^{0} x^{j} ; \\ 0 & \text { otherwise. }\end{cases}
$$

$M$ is constructed directly from the data; it summarizes the relation $R^{0}$. Warshall's algorithm, described in Appendix 2, operates on $M$ to create a matrix $M T$ where

$$
m t_{i j}= \begin{cases}1 & \text { if } x^{i} R x^{j} \\ 0 & \text { otherwise. }\end{cases}
$$

$M T$ can be used to check GARP in the following way.

Algorithm 1: Checking data for consistency with GARP.

Inputs: $\left(p^{i}, x^{i}\right), i=1, \ldots, n$, and the matrix $M T$ representing the relation $R$.

Outputs: whether the data satisfies GARP or not.

1. Is $m t_{i j}=1$ and $p^{j} x^{j}>p^{j} x^{i}$ for some $i$ and $j$ ? If so, we have a violation of GARP.

Algorithm 1 is easily implemented on a computer. According to Afriat's theorem and Fact 1 we can use Algorithm 1 to simply and directly test a finite amount of data with the utility maximization model. If some data satisfies GARP then there is a nice utility function that will rationalize the observed behavior. If the data contains a violation of GARP then there does not exist a nonsatiated utility function that will rationalize the data. Hence we have a straightforward and efficient way to check a finite amount of data for consistency with the neoclassical model of consumer behavior.

\section{RECOVERABILITY_-ORDINAL COMPARISONS OF CONSUMPTION BUNDLES}

Let us turn now to a somewhat different issue, namely the recoverability question described in the introduction. The revealed preference relation $R$ which we discussed in the previous section summarizes all of the preference information contained in the demand observations. Any complete preference ordering that rationalizes the data must contain $R$, and every completion of $R$ that rationalizes the data is a possible preference ordering that generated the data.

However, economists typically assume certain regularity conditions on the allowable preference orderings. For example we might restrict ourselves to preference orderings representable by utility functions that are nonsatiated, 
monotonic, and concave. Afriat's theorem implies that we can always impose such restrictions with no loss of generality; and conversely, that it is impossible to detect violations of these restrictions with a finite amount of demand data.

Suppose then that we are given two new consumption bundles $x^{0}$ and $x^{\prime}$ that have not been previously observed. Suppose that every continuous, nonsatiated, concave, monotonic utility function $u(x)$ that was consistent with $\left(p^{i}, x^{i}\right)$, $i=1, \ldots, n$, implied that $u\left(x^{0}\right)>u\left(x^{\prime}\right)$. Then we might well be justified in concluding that $x^{0}$ was in fact preferred to $x^{\prime}$.

Alternatively we could adopt the following viewpoint. Suppose that every price vector $p^{0}$ at which $x^{0}$ could be demanded-and that was consistent with the data $\left(p^{i}, x^{i}\right), i=1, \ldots, n$-also implied that $x^{0}$ was revealed preferred to $x^{\prime}$. Then certainly we could conclude $x^{0}$ would be preferred to $x^{\prime}$ by any consistent consumer. Let us consider this approach in a bit more detail.

First it is clear that if $x^{0}$ has already been observed-so we know the price at which $x^{0}$ is demanded-there is no problem in verifying whether $x^{0} R x^{\prime}$. Hence we concentrate on the case where $x^{0}$ has not previously been observed. In this case we do not know what price to associate with $x^{0}$ for purposes of the revealed preference comparison. However, we do know what the set of possible prices could be:

DEFINITION: Given any bundle $x^{0}$ not previously observed we define the set of prices that support $x^{0}$ by:

$$
S\left(x^{0}\right)=\left\{p^{0}:\left(p^{i}, x^{i}\right), i=0, \ldots, n, \text { satisfies GARP and } p^{0} x^{0}=1\right\} .
$$

This is simply the set of prices at which $x^{0}$ could be demanded and still be consistent with the previously observed behavior. (The requirement that $p^{0} x^{0}=1$ is a convenient normalization.) We note that Afriat's theorem implies $S\left(x^{0}\right)$ is nonempty for all $x^{0}$-just let $p^{0}$ be the supporting price at $x^{0}$ of any concave utility function that rationalizes the data.

We can use the definition of GARP to provide a convenient description of $S\left(x^{0}\right)$ :

FACT 2: A price vector $p^{0}$ is in $S\left(x^{0}\right)$ if and only if it satisfies the following system of linear inequalities:

$$
\begin{array}{ll}
p^{0} x^{0}=1, & \\
p^{0} x^{0} \leqq p^{0} x^{i} & \text { for all } x^{i} \text { such that } x^{i} R x^{0}, \\
p^{0} x^{0}<p^{0} x^{i} & \text { for all } x^{i} \text { such that } x^{i} P x^{0} .
\end{array}
$$

Proof: Follows immediately from the definition of GARP. 
According to Fact 2, $S\left(x^{0}\right)$ is simply the solution set to a certain system of linear inequalities constructed from the data $\left(p^{i}, x^{i}\right), i=1, \ldots, n$, and the relations $R$ and $P$.

We can use $S(x)$ to describe the set of observations "revealed worse" than $x^{0}$ and "revealed preferred" to $x^{\prime}$ in the following way.

$$
\begin{gathered}
R W\left(x^{0}\right)=\left\{x: \text { for all } p^{0} \text { in } S\left(x^{0}\right), p^{0} x^{0} \geqq p^{0} x^{i}\right. \text { for } \\
\text { some } \left.x^{i} P x \text { or } p^{0} x^{0}>p^{0} x^{i} \text { for some } x^{i} R x\right\}, \\
R P\left(x^{\prime}\right)=\left\{x \text { : for all } p \text { in } S(x), p x \geqq p x^{i}\right. \text { for some } \\
\left.x^{i} P x^{\prime} \text { or } p x>p x^{i} \text { for some } x^{i} R x^{\prime}\right\} .
\end{gathered}
$$

More succinctly, and with only a slight abuse of our earlier definitions, we might write:

$$
\begin{aligned}
& R W\left(x^{0}\right)=\left\{x: \text { for all } p^{0} \text { in } S\left(x^{0}\right), x^{0} P x\right\}, \\
& R P\left(x^{\prime}\right)=\left\{x: \text { for all } p \text { in } S(x), x P x^{\prime}\right\} .
\end{aligned}
$$

These definitions formalize the idea described earlier: if $x^{\prime}$ is in $R W\left(x^{0}\right)$, then whatever the price at which $x^{0}$ is demanded-as long as it is consistent with the previous data - that price will necessarily make $x^{0}$ revealed preferred to $x^{\prime}$. Thus every concave monotonic utility function that rationalizes the data must rank $x^{0}$ ahead of $x^{\prime}$. Of course $R P\left(x^{\prime}\right)$ has a similar interpretation. In fact it is clear from the definitions that $x^{0}$ is "revealed preferred" to $x^{\prime}$ if and only if $x^{\prime}$ is "revealed worse" than $x^{0}$. We record this fact for future reference.

FACT 3: $x^{0}$ is in $R P\left(x^{\prime}\right)$ if and only if $x^{\prime}$ is in $R W\left(x^{0}\right)$.

$R P\left(x^{0}\right)$ and $R W\left(x^{0}\right)$ are extremely important to the rest of our discussion so it is worthwhile presenting a few two-dimensional examples. The simplest casewith one data point-is presented in Figure 2. Let us verify that Figure 2 is correct.

First, we consider $R P\left(x^{0}\right)$. In this simple case, $R P\left(x^{0}\right)$ is simply the convex monotonic hull of all points revealed preferred to $x^{0}$ : namely $x^{1}$ and $x^{0}$ itself. To verify this, let $x$ be any point in $R P\left(x^{0}\right)$, and let $p$ be any (nonnegative) price vector at which $x$ could be demanded. It is geometrically clear that, whatever budget line is chosen, $x$ will be revealed preferred to $x^{0}$-either directly, or indirectly through the observation $x^{1}$. (The reader might check his understanding of this point by indicating the region where $x$ will be directly revealed preferred to $x^{0}$ by all supporting prices, and the region where $x$ will only be indirectly revealed preferred to $x^{0}$ for some supporting prices.) So much for $R P\left(x^{0}\right)$.

In order to verify the construction of $R W\left(x^{0}\right)$, we have to consider all of the prices at which $x^{0}$ could be demanded and still be consistent with the previous 


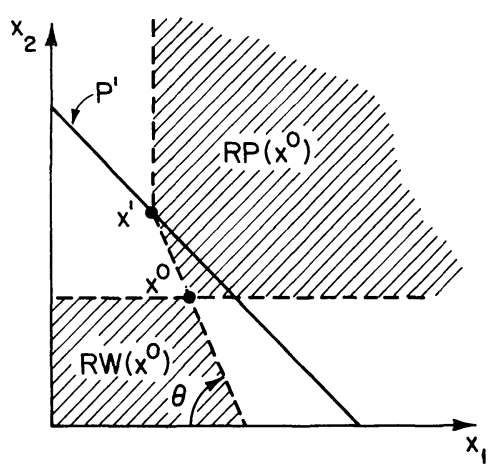

Figure 2.

data point $\left(p^{1}, x^{1}\right)$. In this case GARP imposes an important restriction on $p^{0}$ : the budget line through $x^{0}$ can be no steeper than the indicated angle $\theta$. If it were steeper we would create a violation of GARP: we would have $x^{1} R x^{0}$, and $x^{0} P^{0} x^{1}$. $R W\left(x^{0}\right)$ is the set of points that lie below all budget lines consistent with GARP - exactly as illustrated in Figure 2.

Figure 3 presents a more complex example. As before $R P\left(x^{0}\right)$ turns out to be the convex monotonic hull of all the points revealed preferred to $x^{0} . R W\left(x^{0}\right)$ is a bit more interesting. For all budgets that support $x^{0}$ and satisfy GARP, $x^{0}$ is revealed preferred to $x^{1}$, and a fortiori to all the points beneath $x^{1}$ 's budget set ... including $x^{2}, x^{3}$ and so on.

Now Figure 3 presents us with quite a bit of information about the indifference curve passing through $x^{0}$ : it cannot intersect $R P\left(x^{0}\right)$ or $R W\left(x^{0}\right)$-hence it must lie in between the two. Put another way, the set of bundles preferred to $x^{0}$ (using the true utility function) must always contain $R P\left(x^{0}\right)$, and must be

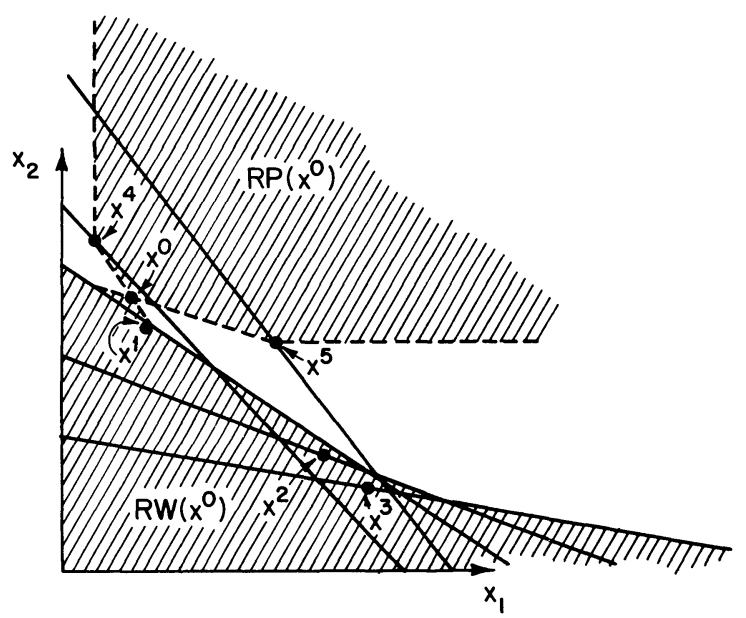

Figure 3. 
contained in the complement of $R W\left(x^{0}\right)$. This last set, the complement of $R W\left(x^{0}\right)$, will be useful later on; we will call it $N R W\left(x^{0}\right)$ for "not revealed worse" than $x^{0}$.

It is clear from Figure 3 that $R P\left(x^{0}\right)$ and $N R W\left(x^{0}\right)$ are not only "inner" and "outer" estimates of the set of bundles preferred to $x^{0}$, they are also the tightest inner and outer estimates. If a point $x^{\prime}$ is not contained in either of these sets then there is a nice utility function that rationalizes the data for which $u\left(x^{0}\right)$ $\geqq u\left(x^{\prime}\right) \ldots$ and there is a nice utility function that rationalizes the data for which $u\left(x^{\prime}\right) \geqq u\left(x^{0}\right)$.

These statements are obvious for the two dimensional example given in Figure 3 , but in fact they are true in general. In order to establish this we need the following criterion for membership in $R W\left(x^{0}\right)$.

FACT 4: $A$ bundle $x^{\prime}$ is in $R W\left(x^{0}\right)$ if and only if there does not exist a $p^{0} \geqq 0$ that satisfies the following system of linear inequalities:

$$
\begin{array}{ll}
p^{0} x^{0}=1, & \\
p^{0} x^{0} \leqq p^{0} x^{i} & \text { for all } x^{i} \text { such that } x^{i} R x^{0}, \\
p^{0} x^{0}<p^{0} x^{i} & \text { for all } x^{i} \text { such that } x^{i} P x^{0}, \\
p^{0} x^{0} \leqq p^{0} x^{j} & \text { for all } x^{j} \text { such that } x^{j} R x^{\prime}, \\
p^{0} x^{0}<p^{0} x^{j} & \text { for all } x^{j} \text { such that } x^{j} P x^{\prime} .
\end{array}
$$

Proof: Suppose $x^{\prime}$ is in $R W\left(x^{0}\right)$. Then any $p^{0}$ that satisfies the first set of inequalities is a supporting price for $x^{0}$ by Fact 2 . By the definition of $R W\left(x^{0}\right)$ it must therefore violate one of the inequalities in the second set.

Conversely suppose $x^{\prime}$ is not in $R W\left(x^{0}\right)$. Then there is some supporting price $p^{0}$ at which $x^{0}$ is not revealed preferred to $x^{\prime}$ by any chain. That is, $p^{0}$ satisfies (2) and (3).

Fact 4 gives us an explicit way to check whether $x^{\prime}$ is revealed worse than $x^{0}$. And by Fact 3 we can see whether $x^{\prime}$ is revealed preferred to $x^{0}$ just by checking whether $x^{0}$ is revealed worse than $x^{\prime}$. Hence we can recover all of the ordinal information in the data by checking whether there exists a solution to a simple set of linear inequalities. This is easily accomplished by solving a simple linear program. Note that the number of constraints in this program will at most be $2 n+1$-and generally be considerably smaller than $2 n+1$.

We can now verify the intuitively plausible statements made earlier concerning the relationship between $R P\left(x^{0}\right), R W\left(x^{0}\right), P\left(x^{0}\right)=\left\{x: u(x)>u\left(x^{0}\right)\right\}$, and $W\left(x^{0}\right)=\left\{x: u\left(x^{0}\right)>u(x)\right\}$.

FACT 5: Let $u(x)$ be any utility function that rationalizes the data. Then for all $x^{0}, R P\left(x^{0}\right) \subset P\left(x^{0}\right) \subset N R W\left(x^{0}\right)$.

Proof: Obvious from the fact that $x^{0} P x^{\prime}$ implies $u\left(x^{0}\right)>u\left(x^{\prime}\right)$ for any utility function that rationalizes the data. 
FACT 6: Suppose that $x^{\prime}$ is not in $R W\left(x^{0}\right)$; then there exists a nonsatiated, continuous, concave monotonic utility function that rationalizes the data for which $u\left(x^{0}\right) \geqq u\left(x^{\prime}\right)$. An analogous statement holds if $x^{\prime}$ is not in $R P\left(x^{0}\right)$.

Proof: Suppose $x^{\prime}$ is not in $R W\left(x^{0}\right)$. Then by Fact 4 there exists a $p^{0}$ supporting $x^{0}$ such that not $x^{0} P x^{\prime}$. Hence by using Fact 16 in Appendix 1, there is a utility function with the stated properties.

FACT 7: Let $x^{0} R x^{\prime}$. Then $R P\left(x^{0}\right) \subset R P\left(x^{\prime}\right)$. Assume further that $x^{\prime}$ is observed as a chosen bundle at some price $p^{\prime}$. Then $R W\left(x^{0}\right) \supset R W\left(x^{\prime}\right)$ and $N R W\left(x^{0}\right)$ $\subset N R W\left(x^{\prime}\right)$.

Proof: Let $\hat{x}$ be in $R P\left(x^{\prime}\right)$. Then for all $\hat{p}$ that support $\hat{x}$ we have $\hat{x} R x^{0}$. Since by hypothesis $x^{0} R x^{\prime}$, transitivity implies $\hat{x} R x^{\prime}$. Hence $\hat{x}$ is in $R P\left(x^{\prime}\right)$.

Let $\hat{x}$ be in $R W\left(x^{\prime}\right)$. Since $x^{\prime}$ is actually chosen at price $p^{\prime}$ this implies $x^{\prime} R \hat{x}$. Since by hypothesis $x^{0} R x^{\prime}$, transitivity implies $x^{0} R \hat{x}$. Hence $\hat{x}$ is in $R W\left(x^{0}\right)$.

\section{RECOVERABILITY - ORDINAL COMPARISONS OF BUDGETS}

In many applications of demand analysis the natural objects of interest are not bundles of goods but are budgets-i.e. prices and expenditures. For example, if one wants to compare proposed changes in the tax structure, it is natural to compare alternative price configurations: given two proposed lists of prices and expenditures $\left(p^{0}, y^{0}\right)$ and $\left(p^{\prime}, y^{\prime}\right)$ we want to know which one is preferred by some individual consumer.

If we had a measure of the consumer's indirect utility function $v(p, y)$ we could simply compute $v\left(p^{0}, y^{0}\right)$ and $v\left(p^{\prime}, y^{\prime}\right)$ and compare the two numbers. If we have only a finite number of observations on a consumer's behavior $\left(p^{i}, x^{i}\right)$, $i=1, \ldots, n$, we could postulate a specification of an indirect utility function, derive the associated demand functions, and estimate the parameters of the resulting demand system. These estimated parameters of the demand system translate directly back to parameters of the indirect utility function which can then be used to make the welfare comparison between the two budgets.

However, the parametric specification necessarily involves an unwarranted maintained hypothesis of functional form. How can we proceed to make a nonparametric comparison of $\left(p^{0}, y^{0}\right)$ versus $\left(p^{\prime}, y^{\prime}\right)$ ?

Let us recall the notion of indirect revealed preference of Sakai [23], Little [18], and Richter [22].

Definition: Given an observed budget $\left(p^{i}, y^{i}\right)$ and a budget $(p, y)$, we say:

(1) $(p, y)$ is directly revealed preferred to $\left(p^{i}, y^{i}\right)$, written $(p, y) R^{0}\left(p^{i}, y^{i}\right)$, if $p x^{i} \leqq y$.

(2) $(p, y)$ is strictly directly revealed preferred to $\left(p^{i}, y^{i}\right)$, written $(p, y) P^{0}$ $\left(p^{i}, y^{i}\right)$, if $p x^{i}<y$. 
(3) $(p, y)$ is revealed preferred to $\left(p^{i}, y^{i}\right)$, written $(p, y) R\left(p^{i}, y^{i}\right)$, if $R$ is the transitive closure of $R^{0}$.

(4) $(p, y)$ is strictly revealed preferred to $\left(p^{i}, y^{i}\right)$, written $(p, y) P\left(p^{i}, y^{i}\right)$ if there exist observed budgets $\left(p^{j}, y^{j}\right)$ and $\left(p^{l}, y^{l}\right)$ such that $(p, y) R\left(p^{j}, y^{j}\right)$, $\left(p^{j}, y^{j}\right) P\left(p^{l}, y^{l}\right),\left(p^{l}, y^{l}\right) R(p, y)$.

Note that the indirect revealed preference relation works exactly opposite to the way the revealed preference relation works. To tell whether $x^{0}$ is revealed preferred to something we need to know the price $p^{0}$ at which $x^{0}$ is demandedand then $x^{0}$ is revealed preferred to the infinite number of bundles beneath its budget line. To tell whether $\left(p^{0}, y^{0}\right)$ is revealed worse than some budget we need to know the bundle $x^{0}$ that is demanded at $\left(p^{0}, y^{0}\right)$-and then $\left(p^{0}, y^{0}\right)$ is revealed worse than the infinite number of budgets $(p, y)$ for which $p x^{0} \leqq y$.

Nevertheless we can apply the same approach to ordinal comparisons to construct dual versions of the results in Section 3. This duality is most clearly exhibited if we normalize prices by dividing through by expenditure so that budgets are uniquely described by $p^{0}=\left(p^{0}, 1\right)$ and $p^{\prime}=\left(p^{\prime}, 1\right)$.

Definition: Given any price $p^{0}$ not previously observed we define the set of bundles that support $p^{0}$ by:

$$
S\left(p^{0}\right)=\left\{x^{0}:\left(p^{i}, x^{i}\right), i=0, \ldots, n, \text { satisfies GARP and } p^{0} x^{0}=1\right\} .
$$

As before the requirement that $p^{0} x^{0}=1$ is only a normalization.

We can now describe the set of budgets "revealed preferred" or "revealed worse" than a given budget by:

$$
\begin{gathered}
R W\left(p^{0}\right)=\left\{p: \text { for all } x \text { in } S(p), 1 \geqq p^{0} x^{i} \text { for some } p^{i} P p,\right. \\
\text { or } \left.1>p^{0} x^{i} \text { for some } p^{i} R p\right\}, \\
R P\left(p^{\prime}\right)=\left\{p: \text { for all } x^{\prime} \text { in } S\left(p^{\prime}\right), 1 \geqq p x^{i} \text { for some } p^{i} P p^{\prime}\right. \\
\text { or } \left.1>p x^{i} \text { for some } p^{i} R x^{\prime}\right\} .
\end{gathered}
$$

Of course these definitions could also be stated as:

$$
\begin{gathered}
R W\left(p^{0}\right)=\left\{p: \text { for all } x \text { in } S(p), 1 \geqq p^{0} x^{i} \text { for some } x^{i} P x,\right. \\
\text { or } \left.1>p^{0} x^{i} \text { for some } x^{i} R x\right\}, \\
R P\left(p^{\prime}\right)=\left\{p: \text { for all } x^{\prime} \text { in } S\left(p^{\prime}\right), 1 \geqq p x^{i} \text { for some } x^{i} P x^{\prime}\right. \\
\text { or } \left.1>p x^{i} \text { for some } x^{i} R x^{\prime}\right\} .
\end{gathered}
$$


Or even more succinctly:

$$
\begin{aligned}
& R W\left(p^{0}\right)=\left\{p \text { : for some } x \text { in } S(p), p^{0} R p\right\}, \\
& R P\left(p^{\prime}\right)=\left\{p \text { : for some } x^{\prime} \text { in } S\left(p^{\prime}\right), p P p^{\prime}\right\} .
\end{aligned}
$$

We can now state the dual versions of Facts 2 and 4. The proofs are completely analogous and are left to the reader.

FACT 8: A bundle $x^{0}$ is in $S\left(p^{0}\right)$ if and only if it satisfies the following system of linear inequalities:

$$
\begin{aligned}
& p^{0} x^{0}=1, \\
& p^{i} x^{i} \leqq p^{i} x^{0} \quad \text { for all } p^{i} \text { such that } p^{0} R p^{i}, \\
& p^{i} x^{i}<p^{i} x^{0} \quad \text { for all } p^{i} \text { such that } p^{0} P p^{i} .
\end{aligned}
$$

FACT 9: $A$ budget $p^{\prime}$ is in $R P\left(p^{0}\right)$ if and only if there does not exist an $x^{0} \geqq 0$ that satisfies the following system of linear inequalities:

$$
\begin{array}{ll}
p^{0} x^{0}=1, & \\
p^{i} x^{i} \leqq p^{i} x^{0} & \text { for all } p^{i} \text { such that } p^{0} R p^{i}, \\
p^{i} x^{i}<p^{i} x^{0} & \text { for all } p^{i} \text { such that } p^{0} P p^{i}, \\
p^{j} x^{j} \leqq p^{j} x^{0} & \text { for all } p^{j} \text { such that } p^{\prime} R p^{j}, \\
p^{j} x^{j}<p^{j} x^{0} & \text { for all } p^{j} \text { such that } p^{\prime} P p^{j} .
\end{array}
$$

Of course the dual versions of Facts 3, 5, and 6 are also true. The statement and proofs of these are left to the reader as well.

Another type of comparison that is often useful is to be able to compare bundles with budgets and vice versa. For example if we are given a direct and an associated normalized indirect utility function, $u(x)$ and $v(p)$, we could consider:

(1) All budgets $p$ preferred to a bundle $x^{0}$ :

$$
P P\left(x^{0}\right)=\left\{p: v(p)>u\left(x^{0}\right)\right\} .
$$

(2) All budgets $p$ worse than a bundle $x^{0}$ :

$$
P W\left(x^{0}\right)=\left\{p: v(p)<u\left(x^{0}\right)\right\} .
$$

(3) All bundles $x$ preferred to a budget $p^{0}$ :

$$
X P\left(p^{0}\right)=\left\{x: u(x)>v\left(p^{0}\right)\right\} .
$$


(4) All bundles $x$ worse than a budget $p^{0}$ :

$$
X W\left(p^{0}\right)=\left\{x: u(x)<v\left(p^{0}\right)\right\}
$$

Each of these constructs has its "revealed preferred" and "revealed worse" analogy:

(1) All budgets $p$ revealed preferred to a bundle $x^{0}$ :

$$
P R P\left(x^{0}\right)=\left\{p: \text { for all } x \text { in } S(p), x P x^{0}\right\} .
$$

(2) All budgets $p$ revealed worse than a bundle $x^{0}$ :

$$
P R W\left(x^{0}\right)=\left\{p: \text { for all } p^{0} \text { in } S\left(x^{0}\right) \text {, and all } x \text { in } S(p), x^{0} P x\right\} .
$$

(3) All bundles $x$ revealed preferred to a budget $p^{0}$ :

$$
X R P\left(p^{0}\right)=\left\{x: \text { for all } p \text { in } S(x) \text {, and all } x^{0} \text { in } S\left(p^{0}\right), x P x^{0}\right\} .
$$

(4) All bundles $x$ revealed worse than a budget $p^{0}$ :

$$
X R W\left(p^{0}\right)=\left\{x: \text { for all } x^{0} \text { in } S\left(p^{0}\right), x^{0} P x\right\} .
$$

If we want to verify whether $p^{\prime}$ is in $P R P\left(x^{0}\right)$, etc. we simply have to write down the associated system of linear inequalities following the general model of Facts 2 and 4. In cases (2) and (4) above, these systems involve unknown $p$ 's and unknown $x$ 's and are therefore somewhat involved. Cases (1) and (4) on the other hand are rather simple. We record this fact for future reference.

\section{FACT 10:}

$$
\begin{aligned}
& P R P\left(x^{0}\right)=\left\{p: 1>p x^{i} \text { for some } x^{i} R x^{0} \text { or } 1 \geqq p x^{i} \text { for some } x^{i} P x^{0}\right\}, \\
& X R W\left(p^{0}\right)=\left\{x: 1>p^{0} x^{i} \text { for some } x^{i} R x \text { or } 1 \geqq p x^{i} \text { for some } x^{i} P x\right\} .
\end{aligned}
$$

\section{EXTRAPOLATION_FORECASTING DEMANDED BUNDLES}

Suppose that we have observed choices $\left(p^{i}, x^{i}\right), i=1, \ldots, n$, and that we are given some new budget $\left(p^{0}, 1\right)$ which has not been previously observed. What choice will the consumer make if his choice is to be consistent with the preferences revealed by his previous behavior? What is the best "overestimate" of the demanded bundle at $p^{0}$ ?

It turns out that we have already answered this question: it is simply the set of bundles that support the budget $p^{0}$, namely $S\left(p^{0}\right)$. For $S\left(p^{0}\right)$ is by definition all of the bundles of goods $x^{0}$ which make the data $\left(p^{i}, x^{i}\right), i=0, \ldots, n$, consistent with GARP. It is therefore the tightest overestimate of the demand correspondence at $p^{0}$ : every bundle in $S\left(p^{0}\right)$ could be a chosen bundle at $p^{0}$ and any 


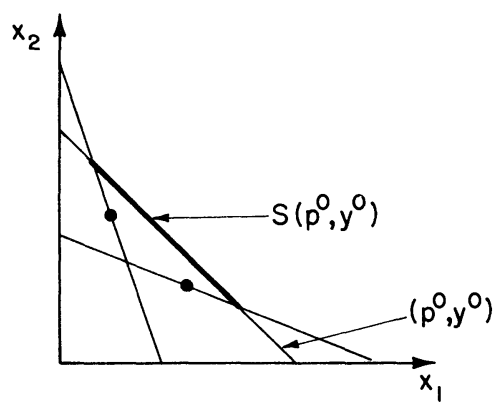

FIGURE 4.

bundle outside of $S\left(p^{0}\right)$ could never be chosen. Figure 4 gives a simple example of $S\left(p^{0}\right)$.

In an analogous manner $S\left(x^{0}\right)$ gives us the tightest overestimate of the inverse demand correspondence.

\section{RECOVERABILITY-BOUNDING A SPECIFIC UTILITY FUNCTION}

It is often desirable to know not only whether some bundle is preferred to some other bundle, but by how much one bundle is preferred to another. Now of course, there is no unique answer to this question: demand theory is completely ordinal in nature and there is no unique cardinal representation of utility. On the other hand it is a common practice to use certain specific cardinalizations of utility in measuring economic welfare.

One particularly useful cardinalization is Samuelson's "money metric" utility function (Samuelson [25]). For reasons that will become apparent, I prefer to call this function the direct income compensation function. We can define it in two equivalent ways:

$$
\begin{aligned}
m\left(p, x^{0}\right)= & \inf p x \\
& \text { such that } x \text { is in } P\left(x^{0}\right)
\end{aligned}
$$

where $P\left(x^{0}\right)=\left\{x: u(x)>u\left(x^{0}\right)\right\}$ or,

$$
m\left(p, x^{0}\right)=e\left(p, u\left(x^{0}\right)\right) .
$$

In the latter definition $e(p, u)$ is the expenditure function and $u(x)$ is the associated utility function. It is obvious from this latter definition that $m\left(p, x^{0}\right)$ behaves like an expenditure function with respect to $p$. It is also straightforward to show that for fixed $p, m\left(p, x^{0}\right)$ behaves like a utility function with respect to $x^{0}$ : since the expenditure function is always increasing in utility, $m\left(p, x^{0}\right)$ is a monotonic transformation of a utility function and is therefore itself a utility function. 
The direct income compensation function can be used to describe at least two measures of "how much" one configuration $\left(p^{0}, x^{0}\right)$ is preferred to another configuration $\left(p^{\prime}, x^{\prime}\right)$, namely Hicks' compensating and equivalent variations:

$$
\begin{aligned}
& C=m\left(p^{\prime}, x^{\prime}\right)-m\left(p^{\prime}, x^{0}\right), \\
& E=m\left(p^{0}, x^{\prime}\right)-m\left(p^{0}, x^{0}\right) .
\end{aligned}
$$

Since $m\left(p^{0}, x\right)$ and $m\left(p^{\prime}, x\right)$ are each utility functions that represent the same preferences, $C$ and $E$ must always have the same sign, but they generally will have different magnitudes.

Let us accept for the moment that $m(p, x)$ is a reasonable cardinalization of utility. The question that then arises is how we might measure it. If we are given a parametric form for the utility function or expenditure function it is always possible to compute $m(p, x)$ directly. However, in the spirit of the nonparametric approach to demand analysis we ask how we might compare functions that provide bounds on $m(p, x)$ that are consistent with a finite set of observed demands $\left(p^{i}, x^{i}\right), i=1, \ldots, n$.

In Section 2 we described the best inner and outer approximations to $P\left(x^{0}\right)$. It is natural to define the upper and lower bounds on the compensation function by:

$$
\begin{aligned}
m^{+}\left(p, x^{0}\right)= & \inf p x \\
& \text { such that } x \text { is in } \operatorname{RP}\left(x^{0}\right), \\
m^{-}\left(p, x^{0}\right)= & \inf p x \\
& \text { such that } x \text { is in } N R W\left(x^{0}\right) .
\end{aligned}
$$

I refer to these as the overcompensation and the undercompensation functions respectively.

FACT 11: Let $m^{+}$and $m^{-}$be defined as above. Then

$$
\begin{aligned}
& m^{+}\left(p^{0}, x\right) \geqq m\left(p^{0}, x\right) \geqq m^{-}\left(p^{0}, x\right) \text { for all } p^{0}, x . \\
& x^{i} R x \text { implies } m^{+}\left(p^{0}, x^{i}\right) \geqq m^{+}\left(p^{0}, x\right) \text {. If } x^{i} R x^{j} \text { and } x^{j} \\
& \text { is chosen at some price } p^{j}, \text { then } m^{-}\left(p^{0}, x^{i}\right) \geqq m^{-}\left(p^{0}, x^{j}\right) .
\end{aligned}
$$

Proof: (i) Follows from Fact 5. (ii) Follows from Fact 7.

Fact 11 shows that: (i) $\mathrm{m}^{+}(p, x)$ and $\mathrm{m}^{-}(p, x)$ do bound the compensation function, and (ii) they are themselves utility functions that respect the revealed preference ordering. 
Thus the overcompensation and undercompensation functions provide theoretically ideal bounds to the compensation function. The problem with these two functions is that they are rather difficult to compute in practice. Recall that Fact 4 gave us a way to verify whether any given bundle $x$ was an element of $R P\left(x^{0}\right)$ or $R W\left(x^{0}\right)$. However, I do not currently have any explicit description of these two sets of the sort suitable for mathematical programming techniques. So instead I have proceeded by defining two approximations to the overcompensation and undercompensation functions. These two approximations do provide bounds, but they are just not the theoretically tightest bounds. We turn now to a description of these approximations.

Let us define the convex, monotonic hull of $\left\{x^{i}: x^{i} R x^{0}\right\}$ :

$$
C M\left(x^{0}\right)=\text { interior of convex hull of }\left\{x: x \geqq x^{i}, x^{i} R x^{0}\right\} \text {. }
$$

FACT 12: $R P\left(x^{0}\right) \supset C M\left(x^{0}\right)$ for all $x^{0}$.

Proof: Let $x$ be a point in $C M\left(x^{0}\right)$ and let $p$ be any price vector that supports $x$. Then I claim $p x>p x^{i}$ for some $x^{i} R x^{0}$. For if not, $p$ would separate $x$ from $C M\left(x^{0}\right)$, a contradiction. Since $x R x^{i}, x^{i} R x^{0}$ we have that $x$ is in $R P\left(x^{0}\right)$.

Then we can define the approximate overcompensation function by:

$$
\begin{aligned}
{a m^{+}}^{+}\left(p, x^{0}\right)= & \inf p x \\
& \text { such that } x \text { is in } \operatorname{CM}\left(x^{0}\right) .
\end{aligned}
$$

Since $C M\left(x^{0}\right)$ is a convex polytope whose vertices are precisely those $x^{i} R x^{0}$, we can also describe this minimization problem by:

$$
a m^{+}\left(p, x^{0}\right)=\min p x^{i}, \quad \text { such that } x^{i} R x^{0} .
$$

Note that this function is quite simple to compute. Nevertheless, this approximate overcompensation function does share some desirable properties with the true overcompensation function.

\section{FACT 13:}

$$
\begin{aligned}
& \operatorname{am}^{+}(p, x) \geqq m^{+}(p, x) \geqq m(p, x) . \\
& x^{0} R x \text { implies am }{ }^{+}\left(p, x^{0}\right) \geqq a m^{+}(p, x) .
\end{aligned}
$$

There exists a convex monotonic preference order $\gtrsim$ such that $\operatorname{am}^{+}\left(p, x^{0}\right)=m\left(p, x^{0}\right)$ for all $x^{0}$.

Proof: The first two parts are obvious. The third is rather detailed. First we define the order and verify that it works; then we establish its properties. 
Let $x \gtrsim x^{\prime}$ if and only if $\mathrm{am}^{+}(p, x) \geqq a m^{+}\left(p, x^{\prime}\right)$. Let us show that the compensation function that goes along with this order is in fact equal to $\mathrm{am}^{+}(p, x)$.

Let $p x^{*}$ solve:

$$
\begin{aligned}
p x^{*}=m(p, \bar{x})= & \min p x \\
& \text { such that } \mathrm{am}^{+}(p, x) \geqq a m^{+}(p, \bar{x})
\end{aligned}
$$

and let $p \tilde{x}$ solve

$$
p \tilde{x}=a m^{+}(p, \bar{x})=\min p x^{i}, \quad \text { such that } x^{i} R \bar{x} .
$$

Now $\tilde{x} R \bar{x}$ so property (2) shows that $\mathrm{am}^{+}(p, \tilde{x}) \geqq a m^{+}(p, \bar{x})$. Hence $\tilde{x}$ is feasible for the first problem and therefore $p x^{*} \leqq p \tilde{x}$.

On the other hand

$$
p x^{*} \geqq a m^{+}\left(p, x^{*}\right) \geqq a m^{+}(p, \bar{x})=p \tilde{x} .
$$

Next we examine the properties of the preference ordering $\gtrsim$.

(a) $\left\{x: \mathrm{am}^{+}(p, x) \geqq k\right\}$ is convex. To prove this, we suppose $\mathrm{am}^{+}\left(p, x^{\prime}\right) \geqq k$ and $\mathrm{am}^{+}\left(p, x^{\prime \prime}\right) \geqq k$. Let

$$
\begin{aligned}
A & =\left\{x^{i}: x^{i} R x^{\prime}\right\}, \\
B & =\left\{x^{i}: x^{i} R x^{\prime \prime}\right\}, \\
C & =\left\{x^{i}: x^{i} R\left(t x^{\prime}+(1-t) x^{\prime \prime}\right)\right\} \quad \text { for some } t \text { such that } 0 \leqq t \leqq 1 .
\end{aligned}
$$

I claim that if $x^{i}$ is in $C$, then $x^{i}$ is in $A \cup B$. For to say $x^{i}$ is in $C$ is to say that there exists a finite sequence such that:

$$
\begin{aligned}
& p^{i} x^{i} \geqq p^{i} x^{r}, \\
& p^{r} x^{r} \geqq p^{r} x^{s}, \\
& p^{l} x^{l} \geqq p^{l}\left(t x^{\prime}+(1-t) x^{\prime \prime}\right) .
\end{aligned}
$$

From the last inequality it is easy to show that either $p^{l} x^{l} \geqq p^{l} x^{\prime}$ or $p^{l} x^{l} \geqq p^{l} x^{\prime \prime}$, which establishes the claim.

Now, since $C \subset A \cup B$, we have:

$$
k \leqq \min _{x \text { in } A \cup B} p x \leqq \min _{x \text { in } C} p x=a m^{+}\left(p, t x^{\prime}+(1-t) x^{\prime \prime}\right) .
$$

(b) If $x^{\prime} \geqq x^{0}$, then $\mathrm{am}^{+}\left(p, x^{\prime}\right) \geqq a m^{+}\left(p, x^{0}\right)$. This follows since $\left\{x^{i}: x^{i} R x^{\prime}\right\}$ $\subset\left\{x^{i}: x^{i} R x^{0}\right\}$.

Thus $\mathrm{am}^{+}\left(p^{0}, x\right)$ is a utility function that bounds the compensation function and the bound is uniformly tight in the sense that there exists a "nice" preference ordering that actually generates $\mathrm{am}^{+}\left(p^{0}, x\right)$ as its compensation function. 


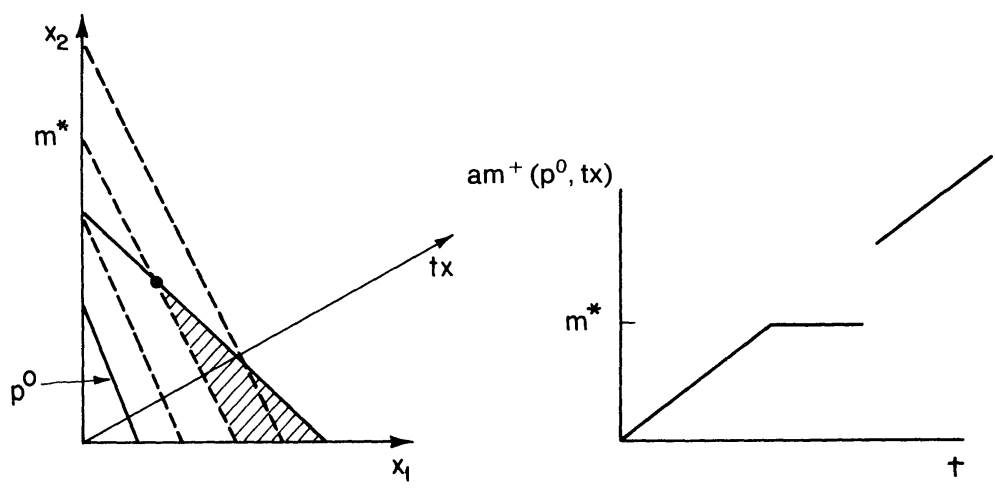

Figure 5.

However it must be pointed out that this ordering typically exhibits regions of satiation, and is in general discontinuous. An example is given in Figure 5. Here all the points in the shaded region are assigned $\mathrm{am}^{+}\left(p^{0}, x\right)=p^{0} x^{1}$. The approximate overcompensation function increases linearly as one moves out the ray $t x$, then is constant, and then jumps discontinuously.

We turn now to the problem of computing an approximation to the undercompensation function. The basic trick here is to get an "inner bound" to $R W\left(x^{0}\right)$ by eliminating the nonconvexities shown in Figure 3 . We define this inner bound by:

$$
I R W\left(x_{0}\right)=\left\{x: \text { for all } p^{0} \text { in } S\left(x^{0}\right), x^{0} R x^{i}, x^{i} \neq x^{0} \text { and } p^{i} x^{i} \geqq p^{i} x\right\} .
$$

The crucial difference between $R W\left(x^{0}\right)$ and $I R W\left(x^{0}\right)$ is the requirement that $x^{i} \neq x^{0}$. This is made clear in Figure 3. The complement of $\operatorname{IR} W\left(x^{0}\right)$, $N I R W\left(x^{0}\right)$, is then given by:

$$
\begin{gathered}
\operatorname{NIRW}\left(x^{0}\right)=\left\{x: p^{i} x>p^{i} x^{i} \text { for some } x^{i} \neq x^{0} \text { such that } x^{0} R x^{i}\right. \\
\text { for all } \left.p^{0} \text { in } S\left(x^{0}\right)\right\} .
\end{gathered}
$$

This is simply a set of a points defined by a finite number of linear inequalities. Hence there is no problem in computing the "approximate undercompensation function":

$$
\begin{aligned}
a m^{-}\left(p^{0}, \bar{x}\right)= & \inf p^{0} x \\
& \text { such that } x \text { is in } \operatorname{NIRW}(\bar{x}) .
\end{aligned}
$$

This also shares some desirable features with the true undercompensation function: 
FACT 14:

$$
\begin{aligned}
& m(p, x) \geqq m^{-}(p, x) \geqq a m^{-}(p, x), \\
& x^{0} R x^{j} \quad \text { implies } \quad a m^{-}\left(p, x^{0}\right) \geqq a m^{-}\left(p, x^{j}\right) .
\end{aligned}
$$

Proof: Left to the reader.

Thus $\mathrm{am}^{-}(p, x)$ bounds the true undercompensation function and it respects the revealed preference ordering, although it does not provide the theoretically ideal bound.

\section{RECOVERABILITY-BOUNDING A SPECIFIC \\ INDIRECT UTILITY FUNCTION}

It is natural to extend the results of the last section to indirect utility comparisons. The function one wishes to bound is the indirect income compensation function

$$
\mu(q ; p, y) \equiv e(q, v(p, y))
$$

where $e(q, u)$ is the expenditure function and $v(p, y)$ is the indirect utility function..$^{5}$ An equivalent way to define $\mu(q ; p, y)$ is:

$$
\begin{aligned}
\mu(q ; p, y)= & \inf q x \\
& \text { such that } x \text { is in } X P(p, y)=\{x: u(x)>v(p, y)\} .
\end{aligned}
$$

Applying the approach of the last section, it appears natural to define the indirect overcompensation function and the indirect undercompensation function by:

$$
\begin{aligned}
\mu^{+}(q ; p, y)= & \inf q x \\
& \text { such that } x \text { is in } \operatorname{XRP}(p, y), \\
\mu^{-}(q ; p, y)= & \inf q x \\
& \text { such that } x \text { is in } N X R W(p, y) .
\end{aligned}
$$

Recall that $X R P(p, y)$ consists of all bundles revealed preferred to the budget $(p, y)$, and $N X R W(p, y)$ consists of all bundles not revealed worse than the budget $(p, y)$; formal definitions were given in Section 3.

It is by now straightforward to verify the following fact:

\footnotetext{
${ }^{5}$ The indirect compensation function was first discussed by McKenzie [19]. It has been extensively treated by Hurwicz and Uzawa [13].
} 
FACT 15: The indirect over and under compensation functions have the following properties:

$$
\mu^{-}(q ; p, y) \leqq \mu(q ; p, y) \leqq \mu^{+}(q ; p, y) .
$$

(ii)

$$
\left(p^{0}, y^{0}\right) R\left(p^{\prime}, y^{\prime}\right) \quad \text { implies } \quad \mu^{-}\left(q ; p^{0}, y^{0}\right) \geqq \mu^{-}\left(q ; p^{\prime}, y^{\prime}\right) \text {. }
$$

If $\left(p^{0}, y^{0}\right)$ is the budget for some observed choice

$$
\text { then } \mu^{+}\left(q ; p^{0}, y^{0}\right) \geqq \mu^{+}\left(q ; p^{\prime}, y^{\prime}\right) \text {. }
$$

Let us now consider the computability of $\mu^{+}$and $\mu^{-}$. As before, we can verify whether any given $x^{\prime}$ is an element of $\operatorname{XRP}(p, y)$ by solving a set of linear inequalities; however it seems difficult to get an explicit description of the sort necessary for mathematical programming.

I therefore suggest the following approximation to $\mu^{+}$:

$$
\begin{aligned}
a \mu^{+}(q ; p, y)= & a m^{+}\left(q, x^{i}\right) \\
& \text { if }(p, y)=\left(p^{i}, y^{i}\right) \text { for some observed }\left(p^{i}, y^{i}\right), \\
= & \max q x \\
& \text { such that } x \text { is in } S(p, y) \text { otherwise. }
\end{aligned}
$$

That is, if $(p, y)$ is observed, we use the value of the approximate overcompensation function. Otherwise, we adopt the most conservative estimate and set $a \mu^{+}(q ; p, y)$ equal to the maximum expenditure over all bundles in the "overestimate" of the demand correspondence. This clearly gives an upper bound on the true overcompensation function.

The indirect undercompensation function is, on the other hand, quite simple to compute. Since Fact 10 gives an explicit description of $X R W(p, y)$, as the solution set to a system of linear inequalities, we can simply compute $\mu^{-}(q ; p, y)$ by solving a small linear program. An illustration of $X R W(p, y)$ and $\mu(q ; p, y)$ is given in Figure 6.

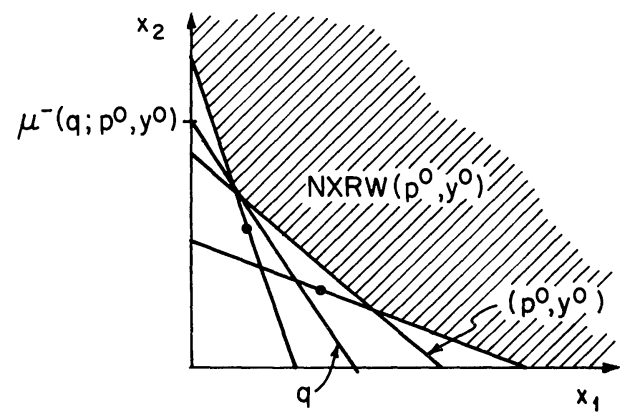

Figure 6. 


\section{SOME APPLICATIONS}

The algorithms described in the previous sections have been assembled in a package of FORTRAN subroutines available from the author. Here I will briefly describe some computational experience with these routines. ${ }^{6}$

First let us consider the issue of testing demand data for consistency with preference maximization. I have applied the routines of Section 1 to several sets of aggregate consumption data. In each case the aggregate consumption data was consistent with GARP: that is, it could have been generated by a single neoclassical "representative consumer." At first glance this may seem somewhat surprising given the negative theoretical results of Sonnenschein [27] and Debreu [8]. However, upon reflection, it is not difficult to understand why this occurs. ${ }^{7}$

Most existing sets of aggregate consumption data are post-war data, and this period has been characterized by small changes in relative prices and large changes in income. Hence, each year has been revealed preferred to the previous years in the sense that it has typically been possible in a given year to purchase the consumption bundles of each of the previous years. Hence no "revealed preference" cycles can occur and the data are consistent with the maximization hypothesis. This observation implies that those studies which have rejected the preference maximization using conventional parametric techniques are rejecting only their particular choice of parametric form.

Given that a set of aggregate consumption data are consistent with preference maximization, we can compute the over- and undercompensation functions described in Sections 5 and 6. One can use these functions to provide some interesting bounds on cost of living indices.

Let $\left(p^{i}, y^{i}\right)$ be a budget in year $i$ and $\left(p^{0}, y^{0}\right)$ be a budget in the base year. Then the true cost of living index is defined by:

$$
i=\frac{\mu\left(p^{0} ; p^{i}, y^{i}\right)}{y^{0}} .
$$

The true cost of living index measures how much money one would need in the base year to be as well off as one was in the comparison year expressed as a fraction of base year expenditure. In order to calculate $i$ one needs the indirect income compensation function which is equivalent to requiring complete knowledge of the individual preference ordering over some range.

However, we can use the results of Section 6 to compute upper and lower bounds on $i$ that are consistent with any finite set of data. Table I presents the results of such a computation using U.S. aggregate consumption data by nine categories from 1947-78.

Note the tightness of the bounds. Typically the overestimate is within 15 per

\footnotetext{
${ }^{6}$ Diewert and Parkan [10] discuss their computational experience with some alternative nonparametric techniques.

${ }^{7}$ For another independent recent application of revealed preference methodology to aggregate data see Landsburg [17].
} 
TABLE I

UPPER AND Lower Bound ON TRUe COST OF Living INDEX ${ }^{a}$ (Classical Bounds in Parentheses)

\begin{tabular}{ccc}
\hline Year & Upper Bound & Lower Bound \\
\hline 1947 & .2496 & .1841 \\
1948 & .2666 & .2004 \\
1949 & .2715 & .2024 \\
1950 & .2906 & .2113 \\
1951 & .3107 & .2237 \\
1952 & .3246 & .2401 \\
1953 & .3409 & .2548 \\
1954 & .3497 & .2634 \\
1955 & .3744 & .2886 \\
1956 & .3905 & .3013 \\
1957 & .4096 & .3172 \\
1958 & .4205 & .3324 \\
1959 & .4500 & .3596 \\
1960 & .4682 & .3779 \\
1961 & .4806 & .3903 \\
1962 & .5082 & .4208 \\
1963 & .5342 & .4499 \\
1964 & .5707 & .4865 \\
1965 & .6119 & .5342 \\
1966 & .6581 & .5864 \\
1967 & .6906 & .6089 \\
1968 & .7524 & .6809 \\
1969 & .8089 & .7406 \\
1970 & .8553 & .8104 \\
1971 & .9174 & .8906 \\
1972 & 1.0000 & 1.0000 \\
1973 & 1.0960 & 1.0409 \\
1974 & 1.1900 & $1.0478(0.9496)$ \\
1975 & 1.2994 & $1.0623(1.0466)$ \\
1976 & 1.4354 & 1.1615 \\
1977 & 1.5767 & .2764 \\
1978 & 1.7330 & 1.4404 \\
\hline
\end{tabular}

a Data are U.S. consumption data by 9 categories from the NBER Time Series Database (Tables 2.3 and 2.4). The goods are motor vehicles, furniture, other durables, food, clothing, gasolıne and oil, housing, transportation, and other services.

cent of the underestimate which allows for a fairly tight estimate of the true cost of living. However, the accuracy of the table is slightly misleading in the following sense.

Given only the information contained in the two observations $\left(p^{0}, y^{0}\right)$ and $\left(p^{\prime}, y^{\prime}\right)$ it is possible to construct the classical bounds depicted in Figure 7. Improvements in these bounds are possible only when some budget set from another sample observation intersects the budget set given by $\left(p^{\prime}, y^{\prime}\right)$ as in Figure 8.

Given the nature of the data, these intersections are quite rare, and in fact only occur for two years 1974 and 1975. Again, the lack of variation in the price data 


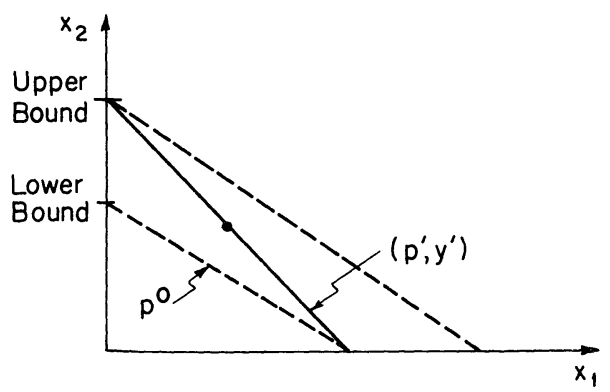

Figure 7.

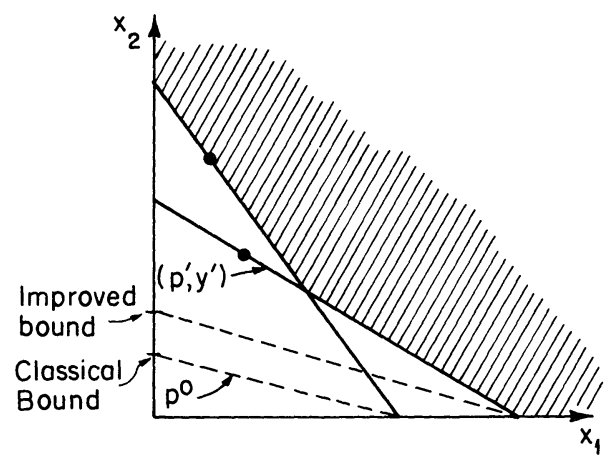

Figure 8 .

limits the power of these methods in this case. However, the techniques proposed here do provide an improvement on the classical bounds when sufficient variation in price data is present.

\section{SUMMARY}

We have shown how the nonparametric techniques of revealed preference analysis can be used to: (1) test a finite amount of data for consistency with preference maximization model; (2) construct a nicely behaved utility function capable of rationalizing a finite amount of demand data; (3) compare previously unobserved consumption bundles and budgets with respect to their ordinal rankings; (4) compute cardinal bounds on the direct and indirect compensation functions; and (5) compute estimates of the direct and indirect demand correspondence consistent with previously observed demand data.

University of Michigan

Manuscript received August, 1980; final revision received August 1, 1981. 


\section{APPENDIX I: A Proof of AFriat's Theorem}

In this appendix we give a proof of Afriat's theorem. The proof we give is based on earlier proofs by Afriat [4] and Diewert [9], but is somewhat more constructive. In fact we will exhibit an algorithm which will actually compute a utility function which rationalizes any given finite amount of data. It turns out that it is convenient to first describe the algorithm to do this computation and then verify that it works in the course of the proof of Afriat's theorem.

The algorithm that we describe below makes use of a subroutine which calculates a maximal element of a finite set with respect to some binary relation.

Let us recall the following definition.

Definition: An element $x^{m}$ of a set $S$ is maximal with respect to a binary relation $B$ if $x^{i} B x^{m}$ implies $x^{m} B x^{t}$.

If $x^{m}$ is a maximal element then either there is nothing that is ranked ahead of it or the only things that are "ahead" of it are things that are indifferent to it.

If we have a finite set with a reflexive and transitive binary relation then there is always at least one maximal element; the following algorithm shows us how to find it. (See Sen [26, p. 11].)

Algorithm 2: Finding a maximal element.

Input: a reflexive and transitive binary relation $B$ defined on a finite set $S=\left(x^{1}, \ldots, x^{n}\right)$ indexed by $I=(1, \ldots, n)$.

Output: an index $m$ where $x^{\prime} B x^{m}$ implies $x^{m} B x^{t}$.

1. Set $m=1, b^{0}=x^{1}$.

2. For each $i=1, \ldots, n$, if $x^{i} B b^{i-1}$ set $b^{i}=x^{i}$, and $m=i$. Otherwise set $b^{i}=b^{i-1}$.

We will let $\max (I)$ be a routine that performs Algorithm 2; that is, given a set $S$ indexed by $I$, $\max (I)$ returns the index of a maximal element in $S$.

It is perhaps not immediately obvious that Algorithm 2 works. Hence we provide the following proof.

FACT 15: The output of Algorithm 2 is the index of a maximal element of $S$.

Proof: First we note that by the transitivity and reflexivity of $B, b^{n} B b^{j}$ for all $j=0, \ldots, n$. Also note that $x^{m}=b^{n}$.

Now suppose we are given some $x^{i} B x^{m}$; i.e. $x^{i} B b^{n}$. We must show that $b^{n} B x^{i}$. First we observe that since $x^{i} B b^{n}$, and $b^{n} B b^{1-1}$, then $x^{\prime} B b^{1-1}$. Line 2 of the algorithm then implies $b^{i}=x^{i}$. But then $b^{n} B b^{l}, b^{l}=x^{l}$ gives $b^{n} B x^{l}$ as required.

We note that the revealed preference relation $R$ is transitive and reflexive, so Algorithm 2 will therefore correctly compute a maximal element. We can now present an algorithm which calculates numbers that satisfy the Afriat inequalities:

Algorithm 3: Constructing the Afriat numbers.

Input: A set of demand observations $\left(p^{l}, x^{l}\right), i=1, \ldots, n$, and the revealed preference relation $R$ that satisfy GARP.

Output: A set of numbers $U^{i}, \lambda^{\prime}>0, i=1, \ldots, n$, that satisfy the Afriat inequalities.

1. $I=\{1, \ldots, n\}, B=\emptyset$.

2. Let $m=\max (I)$.

3. Set $E=\left\{i\right.$ in $\left.I: x^{i} R x^{m}\right\}$. If $B=\emptyset$, set $U^{m}=\lambda^{m}=1$ and go to 6 . Otherwise go to 4 .

4. Set $U^{m}=\min _{i \in E} \min _{j \in B} \min \left\{U^{J}+\lambda^{j} p^{j}\left(x^{i}-x^{j}\right), U^{J}\right\}$.

5. Set $\lambda^{m}=\max _{l \in E} \max _{j \in B} \max \left\{\left(U^{j}-U^{m}\right) / p^{i}\left(x^{j}-x^{i}\right), 1\right\}$.

6. Set $U^{i}=U^{m}, \lambda^{i}=\lambda^{m}$ for all $i \in E$.

7. Set $I=I \backslash E, B=B \cup E$. If $I=\varnothing$, stop. Otherwise, go to 2 .

It is not at all obvious that Algorithm 3 does in fact compute numbers that satisfy the Afriat inequalities; however that fact will be verified in the proof of Afriat's theorem. 
AFRIAT'S THEOREM: The following conditions are equivalent:

(1) There exists a nonsatiated utility function that rationalizes the data.

(2) The data satisfies GARP: if $x^{\prime} R x^{J}$, then $p^{J} x^{J} \leqq p^{J} x^{i}$.

(3) There exist numbers $U^{\prime}, \lambda^{i}>0$ such that $U^{\prime} \leqq U^{j}+\lambda^{\prime} p^{J}\left(x^{i}-x^{J}\right)$ for $i, j=1, \ldots, n$.

(4) There exists a nonsatiated, continuous, concave, monotonic utility function that rationalizes the data.

Proof: $(1) \Rightarrow(2)$. Let $u(x)$ rationalize the data. If $p^{i} x^{i} \geqq p^{i} x^{J}$ then $u\left(x^{i}\right) \geqq u\left(x^{J}\right)$ by definition so that $x^{i} R^{0} x^{j}$ implies $u\left(x^{i}\right) \geqq u\left(x^{j}\right)$. If $p^{i} x^{i}>p^{i} x^{j}$ so that $x^{i} P^{0} x^{j}$, then I claim that $u\left(x^{i}\right)>u\left(x^{j}\right)$. If not, then $u\left(x^{l}\right)=u\left(x^{j}\right)$. But by local nonsatiation there is then an $\hat{x}$ such that $p^{l} x^{l}>p^{\prime} \hat{x}$ and $u(\hat{x})>u\left(x^{i}\right)$. But then $u(x)$ could not rationalize the data point $\left(p^{i}, x^{i}\right)$. Hence $x^{i} P^{0} x^{j}$ implies $u\left(x^{i}\right)>u\left(x^{j}\right)$, and GARP follows.

$(2) \Rightarrow(3)$. In order to prove this we need to verify that Algorithm 3 works; i.e., that the numbers it calculates do indeed satisfy the Afriat inequalities.

At each pass through the algorithm a set of indices of "equivalent" elements, $E$, is removed from $I$ and added to $B$, a set of indices of "better" elements. We will show that after step 6 is executed, the $U$ 's and the $\lambda$ 's at that stage satisfy the Afriat inequalities for all the $U$ 's and $\lambda$ 's calculated up to that point. That is, we will verify the following three statements:

$$
\begin{array}{ll}
U^{\prime} \leqq U^{J}+\lambda^{J} p^{J}\left(x^{\imath}-x^{J}\right) & \text { for all } j \text { in } B \text { and all } i \text { in } E, \\
U^{J} \leqq U^{i}+\lambda^{i} p^{i}\left(x^{J}-x^{\prime}\right) & \text { for all } j \text { in } B \text { and all } i \text { in } E, \\
U^{\imath} \leqq U^{J}+\lambda^{J} p^{J}\left(x^{\imath}-x^{J}\right) & \text { for all } i \text { and } j \text { in } E .
\end{array}
$$

Proof of $(a)$ : By step 4 of the algorithm:

$$
U^{i}=U^{m} \leqq U^{J}+\lambda^{j} p^{J}\left(x^{i}-x^{j}\right) \quad \text { for all } j \text { in } B \text { and all } i \text { in } E \text {. }
$$

Proof of $(b)$ : First note that when the algorithm correctly executes statement $5, p^{i}\left(x^{J}-x^{i}\right)>0$, for all $j$ in $B$. If not, $x^{i} R x^{J}$ for some $j$ in $B$. But then $i$ would have been moved into $B$ before $j$ was moved into $B$.

Hence, the division is well defined and

$$
\lambda^{i}=\lambda^{m} \geqq \frac{U^{J}-U^{i}}{p^{i}\left(x^{J}-x^{i}\right)} \quad \text { for all } j \text { in } B \text { and all } i \text { in } E .
$$

Cross multiplying:

$$
\lambda^{i} p^{\prime}\left(x^{J}-x^{i}\right) \geqq U^{J}-U^{t} \quad \text { for all } j \text { in } B \text { and all } i \text { in } E
$$

which proves (b).

Proof of $(c)$ : First note that $i, j$ in $E$ implies $p^{j}\left(x^{i}-x^{j}\right) \geqq 0$. If not $x^{j} P^{0} x^{i}$, giving a violation of GARP. Now for all $i$ and $j$ in $E$ :

$$
U^{\prime}=U^{J} \quad \text { and } \quad \lambda^{j}=\lambda^{m}>0
$$

so

$$
U^{\prime} \leqq U^{j}+\lambda^{j} p^{j}\left(x^{\prime}-x^{j}\right)
$$

(3) $\Rightarrow(4)$. We define the function $U(x)$ by

$$
U(x)=\min _{i}\left\{U^{t}+\lambda^{t} p^{i}\left(x-x^{\imath}\right)\right\} .
$$

It is clear from the definition that this piecewise linear function has the stated properties. Hence we only need to verify that it rationalizes the data. 

then

First we note that $U\left(x^{l}\right)=U^{i}$ for all $i=1, \ldots, n$. For suppose the minimum is attained at $x^{m}$;

$$
U\left(x^{i}\right)=U^{m}+\lambda^{m} p^{m}\left(x^{m}-x^{i}\right) \leqq U^{i}
$$

since $\lambda^{m} p^{m}\left(x^{l}-x^{l}\right)=0$. But if this inequality were ever strict we would violate one of the Afriat inequalities.

Now suppose we are given some $x$ such that $p^{J} x^{J} \geqq p^{J} x$. We must show that $U\left(x^{J}\right) \geqq U(x)$. This follows directly from the following set of inequalities:

$$
\begin{aligned}
U(x) & =\min _{i}\left\{U^{\prime}+\lambda^{i} p^{\prime}\left(x-x^{l}\right)\right\} \\
& \leqq U^{j}+\lambda^{J} p^{J}\left(x-x^{J}\right) \\
& \leqq U^{J}=U\left(x^{J}\right)
\end{aligned}
$$

since $\lambda^{J} p^{J}\left(x-x^{j}\right) \leqq 0$.

$(4) \Rightarrow(1)$. This is obvious.

It is worthwhile giving a somewhat more heuristic argument for Afriat's Theorem, which more directly exhibits the meaning of the Afriat inequalities. Suppose that we have a differentiable concave utility function that rationalizes some data $\left(p^{i}, x^{i}\right), i=1, \ldots, n$. Then concavity implies

$$
u\left(x^{i}\right) \leqq u\left(x^{j}\right)+D u\left(x^{\jmath}\right)\left(x^{i}-x^{j}\right)
$$

and utility maximization implies

$$
D u\left(x^{J}\right)=\lambda^{j} p^{J} .
$$

Putting these together we see that the Afriat conditions are a necessary condition for utility maximization in this differentiable framework. To motivate the sufficiency result we simply note that by concavity we have $n$ overestimates of the utility at some point $x$ since

$$
u(x) \leqq u\left(x^{l}\right)+\lambda^{i} p^{\imath}\left(x-x^{l}\right) \quad \text { for } \quad i=1, \ldots, n .
$$

Hence the minimum of the right hand side over all observation $i$ - the lower envelope-should give us a reasonable measure of the utility of $x$.

This interpretation of the $U^{\prime}$ 's as utility levels and the $\lambda^{i}$ 's as the marginal utilities of income was first suggested by Afriat [1] and further elucidated by Diewert and Parkan [10]. Varian [29, 30], has used this sort of argument to derive finite necessary and sufficient conditions for a number of specializations of the utility maximization model.

Finally we give a proof of one last fact concerning Afriat's construction that was stated without proof at one point in the text. If $x^{\prime}$ is not revealed preferred to $x^{J}$, then it is intuitively plausible that there is a nice utility function that rationalizes the data for which $u\left(x^{J}\right) \geqq u\left(x^{i}\right)$. This is verified in the next statement.

FACT 16: If not $x^{\prime} R x^{J}$, then there is a nonsatiated, continuous, concave, monotonic utility function that rationalizes the data for which $u\left(x^{J}\right) \geqq u\left(x^{l}\right)$.

Proof: Simply ensure that $\max (I)$ returns the index $j$ before the index $i$. Line 4 of Algorithm 3 then implies that $u\left(x^{J}\right) \geqq u\left(x^{i}\right)$.

\section{APPENDIX II: Computing the Transitive Closure}

The following discussion concerning the computation of the transitive closure of a relation is taken from Aho and Ullman [6], which in turn is based on Warshall [31]. Their results are very slightly generalized in a way that is useful in some other applications (Varian [29]). 


$$
M=\left[\begin{array}{llll}
1 & 1 & 1 & 0 \\
0 & 1 & 1 & 0 \\
0 & 0 & 1 & 1 \\
0 & 0 & 0 & 1
\end{array}\right]
$$

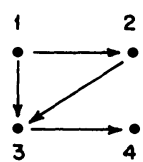

Figure 9.

Let $M$ be an $n$ by $n$ matrix representing a binary relation; i.e. $m_{i j}=1$ if $x^{\prime} R^{0} x^{j}$ and $m_{l j}=0$ otherwise. We can also think of $M$ as representing a directed graph as in Figure 9: there is an arrow from vertex $i$ to vertex $j$ if and only if $m_{i j}=1$. It is this interpretation that gives rise-somewhat indirectly-to Warshall's algorithm.

Suppose now that we have an arbitrary directed graph and some associated cost function $c_{i j}$ where $c_{i j} \geqq 0$ measures the cost of transporting one unit of a good directly from vertex $i$ to vertex $j$. If vertex $i$ and vertex $j$ are not directly connected $c_{l}$ is by definition infinite. Now although the cost of moving $i$ to $j$ directly is given by $c_{i j}$, the cheapest cost of moving $i$ to $j$ may be much less. Warshall's algorithm is concerned with calculating the least cost of moving from any vertex to any other vertex. We denote the magnitude of this least cost by $\bar{c}_{i j}$.

I claim that if we can solve this "least cost problem" we can easily solve the "transitive closure" problem. We just create a cost matrix $C$ where

$$
c_{i j}= \begin{cases}1 & \text { if } m_{i j}=1, \\ \infty & \text { if } m_{i j}=0 .\end{cases}
$$

Now we run $C$ through Warshall's algorithm to compute the least cost matrix $\left(\bar{c}_{i j}\right)$. Then if $\bar{c}_{l j}=l<\infty$ we know that there is some path of length $l$ that connects vertex $i$ with vertex $j$. Hence a method to solve the least cost problem gives us a method to solve the transitive closure problem.

Algorithm 4: Minimum cost of paths in a graph.

Input: $c_{i j}=$ cost of moving from node $i$ to node $j ; c_{i j} \geqq 0$.

Output: $\bar{c}_{i j}=$ minimum cost of moving from node $i$ to node $j$.

(1) Set $k=1$.

(2) For all $i$ and $j$, if $c_{t j} \geqq c_{t k}+c_{k j}$ set $c_{t j}=c_{t k}+c_{k j}$.

(3) If $k<n$, let $k=k+1$ and go to 2 . If $k=n$, set $\bar{c}_{i j}=c_{i j}$ for all $i$ and $j$.

It is not at all obvious that Algorithm 4 does indeed compute the minimum cost of moving from $i$ to $j$ for all $i$ and $j$. But the following argument shows that it works.

FACT 17: Let $(i, l, \ldots, m, j)$ be a path from $i$ to $j$. Then $\bar{c}_{i j} \leqq c_{l l}+\cdots+c_{m j}$.

Proof: Consider the algorithm when it has completed step (2). We will show that $c_{l j}$ is the cost of the cheapest path from $i$ to $j$ that passes through no intermediate vertex with index greater than $k$. This is certainly true for $k=1$, and we suppose it to be true for $k-1$.

Let $(i, l, \ldots, m, j)$ be a path from $i$ to $j$ that passes through no intermediate vertex with index greater than $k$. If it does not pass through vertex $k$ we are done. If it does pass through $k$, we can suppose it only passes through once, since removing a cycle cannot increase the cost. By the induction hypothesis $c_{t k}$ is the cheapest path from $i$ to $k$ with no intermediate vertex greater than $k-1$ and similarly for $c_{k j}$. Since step (2) of the algorithm ensures $c_{i j} \leqq c_{l k}+c_{k j}$, we are done.

Note that step (2) of the algorithm will be executed $n^{3}$ times; thus we can compute the transitive closure of a relation in $n^{3}$ computer additions and comparisons. Of course, if we are using Warshall's algorithm only to compute the transitive closure of a relation we can improve a bit on that bound. Consider for example the following FORTRAN subroutine which computes the transitive closure of a relation represented by the matrix $M$. 
Algorithm 5: Computing the transitive closure.

Input: $M(I, J)=1$ if $p^{i} x^{i} \geqq p^{i} x^{J}, 0$ otherwise. $N=$ number of observations; nobs = maximum number of observations.

Output: $M(I, J)=1$ if $x^{i} R x^{J}, 0$ otherwise.

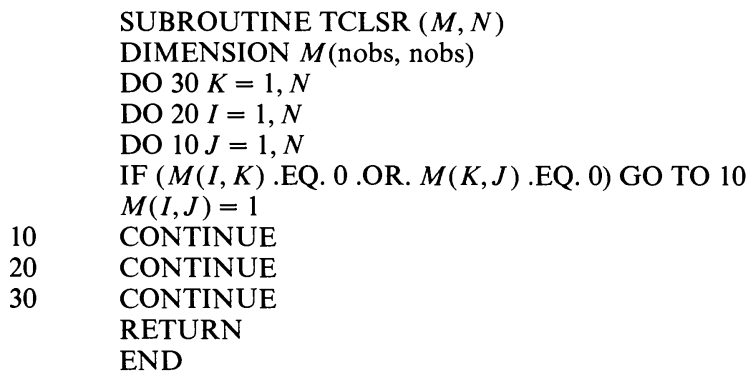

This clearly computes the transitive closure by a straightforward modification of the argument given in Fact 17.

\section{REFERENCES}

[1] Afriat, S.: "The Construction of a Utility Function from Expenditure Data," International Economic Review, 8(1967), 67-77.

[2] - : "The Theory of International Comparison of Real Income and Prices," International Comparisons of Prices and Output, ed. by D. J. Daly. New York: National Bureau of Economic Research, 1972.

[3] — : "On a System of Inequalities on Demand Analysis: An Extension of the Classical Method," International Economic Review, 14(1973), 460-472.

[4] - The Combinatorial Theory of Demand. London: Input-Output Publishing Company, 1976.

[5] —: The Price Index. London: Cambridge University Press, 1977.

[6] Aho, A., And J. Ullman: The Theory of Parsing, Translation, and Compiling, Volume I: Parsing. Englewood Cliffs, New Jersey: Prentice-Hall, Inc., 1972.

[7] Dobell, A.: "A Comment on A. Y. C. Koo's 'An Empirical Test of Revealed Preference Theory'," Econometrica, 33(1965), 451-455.

[8] Debreu, G.: "Excess Demand Functions," Journal of Mathematical Economics, 1(1974), 15-22.

[9] Diewert, E.: "Afriat and Revealed Preference Theory," Review of Economic Studies, 40(1973), 419-426.

[10] Diewert, E., and C. Parkan: "Test for Consistency of Consumer Data and Nonparametric Index Numbers," University of British Columbia, W.P. 78-27, 1978.

[11] Hanoch, G., aNd M. Rothschild: "Testing the Assumptions of Production Theory: A Nonparametric Approach," Journal of Political Economy, 80(1972), 256-275.

[12] Houthakker, H.: "Revealed Preference and the Utility Function," Economica, 17(1950), 159-174.

[13] Hurwicz, L., and H. Uzawa: "On the Integrability of Demand Functions," in Preference Utility and Demand, ed. by J. S. Chipman, et al. New York: Harcourt, Brace, Jovanovich, 1971.

[14] Koo, A.: "An Empirical Test of Revealed Preference Theory," Econometrica, 31(1963), 646-664.

[15] —: "Reply," Econometrica, 32(1965), 456-458.

[16] —-: "Revealed Preference-A Structural Analysis," Econometrica, 39(1971), 89-98.

[17] Landsburg, S.: "Taste Change in the United Kingdom 1900-1955," Journal of Political Economy, 89(1981), 92-104.

[18] LitTle, J.: "Indirect Preferences," Journal of Economic Theory, 20(1979), 182-193.

[19] McKenzie, L.: "Demand Theory Without a Utility Index," Review of Economic Studies, 24(1956), 185-189.

[20] Munroe, I.: "Efficient Determination of the Transitive Closure of a Directed Graph," Information Processing Letters, 1(1971), 56-58. 
[21] Richter, M.: "Revealed Preference Theory," Econometrica, 34(1966), 635-645.

[22] —_: "Duality and Rationality," Journal of Economic Theory, 20(1979), 131-181.

[23] SaKaI, Y.: "Revealed Favorability, Indirect Utility, and Direct Utility," Journal of Economic Theory, 113-129.

[24] Samuelson, P.: "Consumption Theory in Terms of Revealed Preference," Economica, 15(1948), 243-253.

[25] ——: "Complementarity," Journal of Economic Literature, 7(1979), 1255-1289.

[26] Sen, A.: Collective Choice and Social Welfare. San Francisco: Holden-Day, 1970.

[27] Sonnenschein, H.: "Do Walras' Identity and Continuity Characterize the Class of Community Excess Demand Functions?", Journal of Economic Theory, 6(1973), 345-354.

[28] Uebe, G.: "A Note on Anthony Y. C. Koo, 'Revealed Preference-A Structural Analysis'," Econometrica, 40(1972), 771-772.

[29] VARIAN, H.: "Nonparametric Tests of Consumer Behavior," University of Michigan, CREST Working Paper, 1980; forthcoming in Review of Economic Studies.

[30] — : "Nonparametric Tests of Models of Investment Behavior," University of Michigan, CREST Working Paper, 1981.

[31] Warshall, S.: "A Theorem on Boolean Matrices," Journal of the American Association of Computing Machinery, 9(1962), 11-12. 
http://www.jstor.org

\title{
LINKED CITATIONS
}

\author{
- Page 1 of 4 -
}

You have printed the following article:

The Nonparametric Approach to Demand Analysis

Hal R. Varian

Econometrica, Vol. 50, No. 4. (Jul., 1982), pp. 945-973.

Stable URL:

http://links.jstor.org/sici?sici=0012-9682\%28198207\%2950\%3A4\%3C945\%3ATNATDA\%3E2.0.CO\%3B2-O

This article references the following linked citations. If you are trying to access articles from an off-campus location, you may be required to first logon via your library web site to access JSTOR. Please visit your library's website or contact a librarian to learn about options for remote access to JSTOR.

\section{[Footnotes]}

\author{
${ }^{5}$ Demand Theory Without a Utility Index \\ Lionel McKenzie \\ The Review of Economic Studies, Vol. 24, No. 3. (Jun., 1957), pp. 185-189. \\ Stable URL: \\ http://links.jstor.org/sici?sici=0034-6527\%28195706\%2924\%3A3\%3C185\%3ADTWAUI\%3E2.0.CO\%3B2-O
}

${ }^{7}$ Taste Change in the United Kingdom, 1900-1955

Steven E. Landsburg

The Journal of Political Economy, Vol. 89, No. 1. (Feb., 1981), pp. 92-104.

Stable URL:

http://links.jstor.org/sici?sici=0022-3808\%28198102\%2989\%3A1\%3C92\%3ATCITUK\%3E2.0.CO\%3B2-9

\section{References}

${ }^{1}$ The Construction of Utility Functions from Expenditure Data

S. N. Afriat

International Economic Review, Vol. 8, No. 1. (Feb., 1967), pp. 67-77.

Stable URL:

http://links.jstor.org/sici?sici=0020-6598\%28196702\%298\%3A1\%3C67\%3ATCOUFF\%3E2.0.CO\%3B2-K

NOTE: The reference numbering from the original has been maintained in this citation list. 
http://www.jstor.org

\section{LINKED CITATIONS}

- Page 2 of 4 -

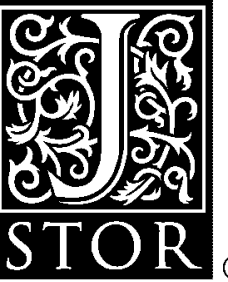

${ }^{3}$ On a System of Inequalities in Demand Analysis: An Extension of the Classical Method S. N. Afriat

International Economic Review, Vol. 14, No. 2. (Jun., 1973), pp. 460-472.

Stable URL:

http://links.jstor.org/sici?sici=0020-6598\%28197306\%2914\%3A2\%3C460\%3AOASOII\%3E2.0.CO\%3B2-K

${ }^{7}$ A Comment on A. Y. C. Koo's "An Empirical Test of Revealed Preference Theory" A. R. Dobell

Econometrica, Vol. 33, No. 2. (Apr., 1965), pp. 451-455.

Stable URL:

http://links.jstor.org/sici?sici=0012-9682\%28196504\%2933\%3A2\%3C451\%3AACOAYC\%3E2.0.CO\%3B2-V

\section{${ }^{9}$ Afriat and Revealed Preference Theory}

W. E. Diewert

The Review of Economic Studies, Vol. 40, No. 3. (Jul., 1973), pp. 419-425.

Stable URL:

http://links.jstor.org/sici?sici=0034-6527\%28197307\%2940\%3A3\%3C419\%3AAARPT\%3E2.0.CO\%3B2-9

${ }^{11}$ Testing the Assumptions of Production Theory: A Nonparametric Approach Giora Hanoch; Michael Rothschild

The Journal of Political Economy, Vol. 80, No. 2. (Mar. - Apr., 1972), pp. 256-275.

Stable URL:

http://links.jstor.org/sici?sici=0022-3808\%28197203\%2F04\%2980\%3A2\%3C256\%3ATTAOPT\%3E2.0.CO\%3B2-8

\section{${ }^{12}$ Revealed Preference and the Utility Function}

H. S. Houthakker

Economica, New Series, Vol. 17, No. 66. (May, 1950), pp. 159-174.

Stable URL:

http://links.jstor.org/sici?sici=0013-0427\%28195005\%292\%3A17\%3A66\%3C159\%3ARPATUF\%3E2.0.CO\%3B2-J

\section{${ }^{14}$ An Empirical Test of Revealed Preference Theory}

Anthony Y. C. Koo

Econometrica, Vol. 31, No. 4. (Oct., 1963), pp. 646-664.

Stable URL:

http://links.jstor.org/sici?sici=0012-9682\%28196310\%2931\%3A4\%3C646\%3AAETORP\%3E2.0.CO\%3B2-X

NOTE: The reference numbering from the original has been maintained in this citation list. 
http://www.jstor.org

\section{LINKED CITATIONS}

- Page 3 of 4 -

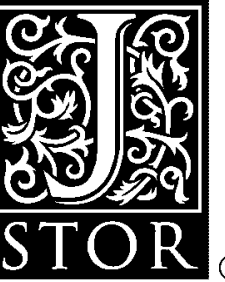

${ }^{15}$ A Comment on A. Y. C. Koo's "An Empirical Test of Revealed Preference Theory": Reply Anthony Y. C. Koo

Econometrica, Vol. 33, No. 2. (Apr., 1965), pp. 456-458.

Stable URL:

http://links.jstor.org/sici?sici=0012-9682\%28196504\%2933\%3A2\%3C456\%3AACOAYC\%3E2.0.CO\%3B2-Q

${ }^{16}$ Revealed Preference--A Structural Analysis

Anthony Y. C. Koo

Econometrica, Vol. 39, No. 1. (Jan., 1971), pp. 89-97.

Stable URL:

http://links.jstor.org/sici?sici=0012-9682\%28197101\%2939\%3A1\%3C89\%3ARPSA\%3E2.0.CO\%3B2-G

${ }^{17}$ Taste Change in the United Kingdom, 1900-1955

Steven E. Landsburg

The Journal of Political Economy, Vol. 89, No. 1. (Feb., 1981), pp. 92-104.

Stable URL:

http://links.jstor.org/sici?sici=0022-3808\%28198102\%2989\%3A1\%3C92\%3ATCITUK\%3E2.0.CO\%3B2-9

\section{${ }^{19}$ Demand Theory Without a Utility Index}

Lionel McKenzie

The Review of Economic Studies, Vol. 24, No. 3. (Jun., 1957), pp. 185-189.

Stable URL:

http://links.jstor.org/sici?sici=0034-6527\%28195706\%2924\%3A3\%3C185\%3ADTWAUI\%3E2.0.CO\%3B2-O

\section{${ }^{21}$ Revealed Preference Theory}

Marcel K. Richter

Econometrica, Vol. 34, No. 3. (Jul., 1966), pp. 635-645.

Stable URL:

http://links.jstor.org/sici?sici=0012-9682\%28196607\%2934\%3A3\%3C635\%3ARPT\%3E2.0.CO\%3B2-G

\section{${ }^{24}$ Consumption Theory in Terms of Revealed Preference}

Paul A. Samuelson

Economica, New Series, Vol. 15, No. 60. (Nov., 1948), pp. 243-253.

Stable URL:

http://links.jstor.org/sici?sici=0013-0427\%28194811\%292\%3A15\%3A60\%3C243\%3ACTITOR\%3E2.0.CO\%3B2-0

NOTE: The reference numbering from the original has been maintained in this citation list. 
http://www.jstor.org

\title{
LINKED CITATIONS \\ - Page 4 of 4 -
}

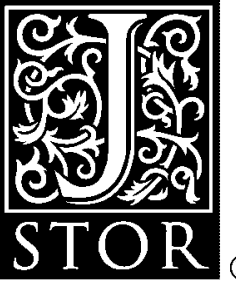

\author{
${ }^{28}$ A Note on Anthony Y. C. Koo, "Revealed Preference--A Structural Analysis" \\ Goetz Uebe \\ Econometrica, Vol. 40, No. 4. (Jul., 1972), p. 771.
}

Stable URL:

http://links.jstor.org/sici?sici=0012-9682\%28197207\%2940\%3A4\%3C771\%3AANOAYC\%3E2.0.CO\%3B2-N

NOTE: The reference numbering from the original has been maintained in this citation list. 